\title{
DE LA IDEOLOGÍA A LA PRÁCTICA: EL CASO DE LA ZONA DE INTERÉS TURÍSTICA DE LA CUENCA DEL LAGO RANCO, CHILE
}

\author{
Esteban Garrido Muñoz \\ Curso Política Pública en Turismo \\ Magister en Gestión e Innovación del Turismo de Intereses Especiales. \\ esgarrido@gmail.com
}

\section{RESUMEN}

La definición y los procesos administrativos relacionados con las Zonas de Interés Turístico (ZOIT) en Chile han variado durante sus cuatro décadas de vigencia. Este ensayo analiza dichas modificaciones utilizando como marco territorial la Cuenca del Lago Ranco, Región de Los Ríos, centrándose especialmente en los actores participantes del proceso de implementación, identificando factores ventajosos para su desarrollo, factores limitantes y desafíos futuros.

PALABRAS CLAVES: ZOIT, zona de interés turístico, Cuenca del Lago Ranco, actores participantes. 


\section{IDEOLOGY INTO PRACTICE: THE CASE OF THE TOURIST ATTRACTIONS OF THE BASIN OF RANCO LAKE, CHILE.}

\section{Esteban Garrido Muñoz}

Curso Política Pública en Turismo

Magister en Gestión e Innovación del Turismo de Intereses Especiales.

esgarrido@gmail.com

\section{RESUMEN}

The definition and administrative processes related to ZOITs in Chile have varied during its four decades of validity. This paper analyzes these modifications using as a territorial framework the Lake Ranco Basin, Los Ríos Region, focusing especially on the actors involved in the implementation process, identifying factors advantageous for their development, limiting factors and future challenges.

KEYWORDS: ZOIT, area tourist attractions, Lake Ranco Basin's, actors involved. 
A fines del año 1975, mientras en Bill Gates fundaba Microsoft y finalizaba la guerra de Vietnam, en Chile se creaba mediante el Decreto Ley N¹224 (Congreso Nacional de Chile, 1975) el Servicio Nacional de Turismo, Sernatur, quien, entre muchas otras cosas, tenía la atribución de declarar de Zonas y Centros de Interés Turístico - ZOIT (Castelblanco Cárdenas, Cerna Cerna, Collao Pardo, \& Donoso Caucamán, 2014). Estas zonas son integradas por aquellos territorios comunales, intercomunales o determinada área dentro de éstos, que tengan condiciones especiales para la atracción turística y que requieren de una planificación integrada para promover las inversiones del sector privado (Congreso Nacional de Chile, 2010). Sin duda, para las administraciones comunales contar con una herramienta de planificación como ésta era atractivo, por lo cual más de 20 fueron declaradas en el período 1979-2009. (Sernatur, 2016).

¿Qué esperaban los equipos comunales de esto? Tal vez el principal motor fue el ego profesional de los equipos locales por conseguir tal logro, sin dejar de lado la espera de beneficios para zonas puntuales que permitieran establecer políticas comunales o regionales de fomento de la actividad turística y que llevara a una planificación complementaria con otros usos de suelo.

Pero ¿cuántas de ellas efectivamente se beneficiaron del procedimiento en esa época? Es difícil decirlo, debido a que no había un formato establecido ni un mecanismo muy estricto que instara a generar y ejecutar un plan de acción asociado a tal declaratoria. Estoshechos, enmarcados enunaseriedesucesosnoexentos depolémica, dan pie para que en el año 2010 se promulgue la nueva Ley de Turismo N²0.432 (Congreso Nacional de Chile, 2010) donde se redefinen las Zonas de Interés Turístico y cuyos requisitos quedan plasmados en el Decreto $\mathrm{N}^{\circ} 172$. El paso de una modalidad a otra es un proceso muy interesante de analizar, en especial cuando son muchos los territorios que se enfrentan a este nuevo procedimiento administrativo. Para ejemplificar, consideremos el caso del territorio Lago Ranco-Futrono, el cual fue declarado Zoit por primera vez en diciembre del año 2009 y nuevamente en enero del 2015.. 
Para tener una mirada global del proceso, un breve resumen de los pasos actuales que un territorio debe recorrer para ser declarado y funcionar como una Zoit sería: a) que una Corporación de derecho privado u organización gremial complete el formulario de postulación; b) El territorio, tras ser evaluado por la Subsecretaría de Turismo, debe generar un Plan de Acción a dos años plazo, cuya responsabilidad de ejecución es de la institución postulante; c) El Plan de Acción es presentado a la Subsecretaría y revisado por el Comité de Ministros de Turismo el cual en caso de aprobación se genera una Declaratoria consistente en un Decreto con Fuerza de Ley; d) La ejecución de las acciones comprometidas en el Plan de Acción, y finalmente e) El seguimiento anual acompañado por Sernatur, y evaluación final de cumplimiento por parte de la Subsecretaría de Turismo.

En el caso específico de la Zoit Lago Ranco-Futrono actualmente se lleva a cabo el proceso de implementación, experiencia de la que emergen una serie de temas críticos, definidos así por cuanto su desarrollo difiere de lo esperado por los equipos locales. Uno de los más relevantes es la naturaleza de la Institución postulante, puesto que marca una diferencia entre el primer proceso Zoit y el segundo. En el primero eran los Municipios junto a Sernatur quienes postulaban sus territorios, mientras en el segundo podían hacerlo sólo agrupaciones o corporaciones de privados.

¿Ventajas? Lo trascendental de la visión de los privados, quienes superan la barrera política temporal de los cuatro años de Gobierno local, pudiendo llegar a trabajar en períodos más largos. Por otra parte cuando son los privados quienes lideran el proceso evidencia que hay un tejido empresarial fortalecido, clave para el desarrollo turístico.

¿Desventajas? Varias, entre las cuales se pueden mencionar: que es difícil medir el nivel de representatividad de la institución postulante, en especial cuando existe más de una en un territorio o cuando ésta está compuesta por algunos tipos de empresas; por otra parte 
la tendencia es que los privados no asuman responsabilidades de desarrollo local, asumiendo que es obligación de los organismos públicos, lo que traduce en un menor cantidad de postulaciones, comparando el primer proceso Zoit con el segundo; otro riesgo que se corre en el hecho que parte de la planificación territorial esté siendo liderada por los intereses de los privados, es que pudiese primar la priorización y gestión pública a los lugares donde solamente existen intereses de estos actores; otro elemento no menor es que existe la posibilidad que existan varios privados postulando simultáneamente a un territorio similar, traslapándose los intereses y los territorios, generando problemas al momento de planificar.

Se especulan algunas modificaciones al Reglamento que enmarca las Zoit, dentro de las que se menciona un retorno al liderazgo liderado por el sector público. Esto es beneficioso por cuanto se subentiende que hay un conocimiento más cabal del territorio, un espíritu de responsabilidad pública y mayores posibilidades de coherencia entre distintos instrumentos de planificación, no obstante se debe tener precaución de dar continuidad a los procesos a pesar de la rotación de las autoridades, propias de un estado democrático.

El caso de Lago Ranco - Futrono considera a dos comunas colindantes, cuyo proceso en su primera etapa fue liderado por ambas Municipalidades y apoyo técnico de Sernatur. En esta segunda etapa el liderazgo estuvo en manos de la Corporación Consejo Privado de la Cuenca del Lago Ranco (Corporación Cuenca del Lago Ranco, 2016), organización que está presente en el territorio y que desarrolla diversas líneas de trabajo en beneficio del turismo. Ella se compone de cerca de $28 \mathrm{em}-$ presarios de distinto tamaño y cuya despliegue territorial considera cinco comunas: Paillaco, La Unión, Río Bueno, Lago Ranco y Futrono

Una particularidad de este territorio fue que desde el año 2006 se comienza a generar alianzas público privadas, las que se vieron fortalecidas con el Programa Chile Emprende (Gobierno Regional Chacabuco, 2009) fruto de la cual nace esta Corporación. Es evidente que todos los involucrados 
tanto públicos como privados, pudieron reconocer los beneficios de trabajar unidos por un territorio en común, lo cual se plasma en la firma de un Convenio de Cooperación entre la Corporación del Ranco y las Municipalidades de Lago Ranco y Futrono para el desarrollo de este proceso.

Éste es un caso particular de gobernanza, el cual queda plasmado en su Plan de Acción. Sin embargo, desde un principio se ha evidenciado que otros actores deben ser incorporados. Tal es el caso de Sernatur, pero no sólo con el rol fiscalizador actual, sino que en un rol de coordinación con otros servicios públicos. La Gobernación y el Consejo de Seremis de Turismo, por su parte, debiesen ser la extensión del Comité de Ministros, involucrándose y sentando las bases para su aplicación y continuidad. Por todo lo anterior, es fundamental que el Reglamento especifique un modelo de gobernanza que permita replicar y mejorar la experiencia del territorio Lago Ranco-Futrono, contemplando al menos los tres componentes: las empresas privadas, las Municipalidades y el Gobierno regional, con el fin de dar una respuesta positiva a una interrogante obvia: con los actuales actores involucrados ¿la Zoit se puede considerar un instrumento de Ordenamiento territorial, consensuado entre el público privado que permita apalancar y gestionar recursos o es meramente una tarjeta de presentación?

Es una pregunta que por el momento no tiene respuesta.

\section{BIBLIOGRAFÍA}

Castelblanco Cárdenas, J., Cerna Cerna, K., Collao Pardo, K., \& Donoso Caucamán, N. (2014). Implentación de la Ley de turismo N²0.423 percepción de actores en la Región de Los Ríos. Valdivia: Universidad Austral de Chile.

Congreso Nacional de Chile. (08 de noviembre de 1975). Biblioteca del Congreso Nacional de Chile. Recuperado el 14 de junio de 2016, de Decreto Ley N¹224 Crea el Servicio Nacional del Turismo: http://bcn. 
Gobierno Regional Chacabuco. (julio de 2009). Gobernación Chacabuco. Recuperado el 29 de junio de 2016, de Chacabuco, un territorio que requiere participar del Programa Chile Emprende: http://www.gobernacionchacabuco.gov.cl/filesapp/chilemprende.pdf

Sernatur. (enero de 2016). Servicio Nacional de Turismo - Gobierno Transparente. Recuperado el 2016 de junio de 13, de Actos y documentos publicados en el Diario Oficial: http://www.sernatur.cl/transparencia/ transparencia/marco-normativo/actos-y-doc.html TVN. (09 de abril de 2014). Ex ministro afirma que ZOIT derogadas no cumplían requisitos. Recuperado el 29 de junio de 2016, de 24 horas.cl: http://www.24horas.cl/economia/ex-ministro-afirma-que-zoit-derogadasno-cumplian-requisitos- 1173270 